\title{
Los albergues para migrantes en México frente al Covid-19: el caso de Mexicali, Baja California
}

\section{Migrant shelters in Mexico facing up Covid-19: the case of Mexicali, Baja California}

\author{
Kenia María Ramírez-Meda y Adriana Teresa Moreno-Gutiérrez \\ Facultad de Ciencias Sociales y Políticas de la Universidad Autónoma \\ de Baja California, México
}

\begin{abstract}
Resumen
El artículo analiza la situación de los albergues que alojan a migrantes en la ciudad fronteriza de Mexicali, Baja California, derivado la suspensión de fondos por parte del gobierno federal a estas organizaciones de la sociedad civil y el posicionamiento de Baja California como uno de los epicentros de la pandemia. La estrategia metodológica es de corte mixto, ya que por medio de la revisión documental se establecen los indicadores que refieren los parámetros mínimos de operación que deben ser adoptados por los albergues ante el Covid-19 para posteriormente elaborar trabajo de campo por medio de entrevistas semiestructuradas a actores clave. En los resultados y conclusiones se evidencia la falta de voluntad política y de mecanismos de coordinación del gobierno hacia estas organizaciones, lo que agudiza la situación de vulnerabilidad de los migrantes.
\end{abstract}

Palabras clave: Albergues, migrantes, Covid-19; México, Baja California.

\begin{abstract}
In this research we seek to demonstrate the situation of the shelters that house migrants in the border city of Mexicali, Baja California, the foregoing derived from two elements that have impacted their operation: the suspension of funds by the government to these organizations of civil society and the positioning of Baja California as one epicenters of the pandemic. The methodological strategy is mixed, through the documentary review we establish the indicators that refer to the minimum operating parameters that must be adopted by the shelters in the face of the Covid-19 to later elaborate field work through semi-structured interviews to key players. In the results and conclusions, we show the lack of political will and government coordination mechanisms towards these organizations, which exacerbates the situation of vulnerability of migrants.
\end{abstract}

Keywords: Shelters, migrants, Covid-19, Mexico, Baja California.

Artículo recibido el 12 de noviembre de 2020 y aprobado el 21 de enero de 2021. 


\section{INTRODUCCIón}

E

n México se presentan dinámicas migratorias de origen, tránsito, destino y retorno. La cercanía con Estados Unidos favorece el tránsito de personas que provienen de la región de Centroamérica. En los 80’s las guerras civiles en Guatemala, El Salvador y Nicaragua, debilitaron las instituciones del Estado, propiciando violaciones de derechos humanos, exclusión y desigualdad social (Devia, Ortega y Mallaganes, 2014; Equipo Regional de Monitoreo y Análisis de Centroamérica, 2019), las cuales se perpetran hasta nuestros días y se acentúan con altos índices de violencia, producto de las estructuras criminales que conducen a la proliferación de pandillas, narcotráfico y entre otros delitos (Devia et al., 2014; Jiménez, 2016) que promueven la expulsión de personas en búsqueda de mejores oportunidades y calidad de vida.

El tránsito de los flujos migratorios por México también se ha estimulado por desastres naturales, por ejemplo, el huracán Mitch en 1998, que "dejó aún más pobres a países como Honduras, Nicaragua, El Salvador y Guatemala" (Carrasco, 2013: 175), como afirma el autor, pocos obtuvieron visas humanitarias en Estados Unidos, por lo que optaron por el ingreso no documentado.

La región de Centroamérica ha colocado a México en uno de los principales países de migración en tránsito irregular o no documentada en América Latina (Martínez, Cobo y Narváez, 2015). Durante el periodo 2010 a 2017 estos eventos se incrementaron, pasando de los 128.4 mil a 296.8 mil, donde al menos 9 de cada 10 personas son centroamericanos (SEGOB y UPM, 2019). Además, el número de mexicanos devueltos por las autoridades de Estados Unidos en 2017 y 2018 aumentaron de 154.6 mil a 192.2 mil (El COLEF et al., 2019).

Las entidades federativas y ciudades fronterizas al norte de México son lugares de tránsito y destino de las migraciones documentadas, irregulares, solicitantes de la condición de refugiado en México, solicitantes de asilo en EE. UU. y flujos masivos que intentan ingresar a a este país. De éstos últimos, en Baja California (BC) entidad ubicada al noroeste de México, debido a las políticas restrictivas de Estados Unidos, cerca de 4 mil migrantes haitianos en 2017 quedaron varados, 
de este número 2,800 se encontraban en proceso de regularización en México (Luna, 2017), del total, el 75 por ciento se albergó en la ciudad de Tijuana y 25 por ciento en Mexicali (Paris, 2018: 9). En el periodo de 2016 a 2019, 623 haitianos tramitaron la tarjeta de visitante por razones humanitarias y 148 acreditaron la solicitud de refugio en Mexicali (Urbina, 2020: 3). A finales de 2018 y 2019 ocurrieron tres caravanas migrantes principalmente de origen centroamericano, quienes pretendían solicitar asilo en Estados Unidos, conformadas aproximadamente por 11,500 personas, de este número en diciembre de 2018 alrededor de 900 personas de las caravanas se encontraban en Mexicali (Albicker et al., 2019).

A mediados del año 2019 se negoció por parte de Marcelo Ebrard Casaubón, Secretario de Relaciones Exteriores de México, que los solicitantes de asilo (provenientes de países centroamericanos) en Estados Unidos permanezcan en espera en territorio mexicano durante su proceso de solicitud ante autoridades migratorias y también su proceso de revisión en la Corte, bajo el programa denominado "Quédate en México" se pusieron en marcha los Protocolos de Protección al Migrante (MPP).

Como respuesta a las caravanas migrantes, el despliegue de estos protocolos obliga a permanecer en México a los migrantes durante su proceso de asilo en Estados Unidos (Avendaño, Siu, Romero y Moreno, 2019). Las devoluciones a México al aplicar los MPP comenzaron en enero del 2019 a Tijuana, en marzo a Mexicali y Ciudad Juárez, en agosto se registraron 38,291 devoluciones en cinco puertos de entrada a Estados Unidos (París y García, 2019: 2), para octubre, en BC se contaron alrededor de 13 mil personas (Avendaño et al., 2019: 5).

\section{Política Migratoria 2012-2020}

Durante el sexenio de Enrique Peña Nieto en México (2012-2018) y la Presidencia de Barack Obama en Estados Unidos (2009-2017), en ambos países se configuró una política migratoria basada en las deportaciones masivas, México se convirtió en el país intermedio más importante para regresar a los migrantes a sus países de origen, luego de ser expulsados del territorio estadounidense. Como parte de la armonización política migratoria mexicana con la de Estados Unidos se creó el programa Frontera Sur mediante el cual se reforzó una política de velar por la seguridad de las fronteras, detenciones masivas de migrantes en tránsito, deportaciones y aumento de agentes migratorios 
en la frontera sur, el objetivo era impedir que cruzaran el territorio nacional hasta llegar a la frontera norte.

Al llegar a la presidencia Andrés Manuel López Obrador en el año 2018 esbozó un discurso que ya había iniciado durante su campaña política en pro de la defensa y protección de los derechos humanos de los migrantes, tanto los mexicanos que residen en Estados Unidos, como aquellos no mexicanos que utilizan a México como país de tránsito con especial énfasis en los centroamericanos. Incluso con la llegada de la primera caravana de migrantes en octubre de 2018 se respondió con la oferta de visas humanitarias y en el mes de enero del año 2019 se implementó el "Programa emergente de emisión de tarjetas de visitante por razones humanitarias" (Ruiz, Tenison y Weyandt, 2020: 26), mismo que finalizó en ese mismo mes, esta iniciativa fue seguida de subsecuentes esfuerzos por otorgar estas tarjetas humanitarias a los centroamericanos para que transitaran por México de una forma más ágil y expedita, en este sentido se reconoce que:

Aunque fue de corta duración, el Programa Emergente reflejó el enfoque humanitario inicial de la administración de López Obrador respecto a la política migratoria. Entre diciembre de 2018 y abril de 2019, el INM otorgó un total de 26,584 visas humanitarias. Este fue un aumento del 7,000 por ciento respecto al año anterior, cuando entre diciembre de $2017 \mathrm{y}$ abril de 2018, el INM sólo otorgó 5,102 visas humanitarias (Ruiz, Tenison y Weyandt, 2020: 28).

El cambio sobrevino cuando se pusieron en marcha los Protocolos de Protección al Migrante y el programa Remain in Mexico, adicional a esto el gobierno inició el despliegue de la Guardia Nacional con especial énfasis en la frontera Sur, con el fin de controlar la entrada y tránsito de los grupos de migrantes que buscan llegar hasta el norte.

El papel de los gobiernos estatales (entidades federativas) ha sido también muy limitado ya que al ser la migración un tema gestionado y regulado por la federación, los recursos que se utilizan para la atención y gestión de estos flujos son determinados y redirigidos por el gobierno federal hacia las arcas estatales y municipales sin tomar en cuenta las verdaderas necesidades ni la intensidad del fenómeno lo que genera una limitada capacidad de acción de los gobiernos locales frente al mismo. El gobierno federal por su parte también construyó dos albergues o centros de integración para migrantes donde se pretendía la atención de los MPP, con limitados resultados en Tijuana y 
Ciudad Juárez y con una actuación que se vio mermada por la pandemia por Covid-19 en la ciudad de Mexicali donde solo operó por seis meses, pero sin ofrecer el servicio de alojamiento, solo de atención y asesoría en materia de regularización, empleo y salud.

Históricamente en México, la sociedad civil ha representado el sector con más incidencia en la atención de los flujos migratorios, específicamente los albergues para migrantes que operan bajo la figura de asociación civil, donde las personas en contexto de migración obtienen refugio, alimento, ropa, atención en materia de salud y algunos otros servicios. Adicional a esto, en los últimos tres años en la ciudad de Mexicali se han sumado una cantidad importante de actores internacionales de tipo gubernamental y no gubernamental para apoyar en la gestión y atención de estos flujos, entre los más importantes se ubican; la Organización Internacional para las Migraciones (OIM), el Alto Comisionado de las Naciones Unidas para los Refugiados (ACNUR), Médicos sin Fronteras (MSF), Save the Children y Border Kindness.

El incremento de los flujos migratorios y las medidas de contención adoptados por los gobiernos de Estados Unidos y México como es el caso de los MPP impactan en la operatividad de los albergues de las ciudades de la frontera norte, debido a la ampliación de los periodos de estancia y aumento del número de servicios que ofrecen. En Mexicali, BC, se cuentan con 11 albergues con capacidad para 1,460 personas (Coubès, Velasco y Contreras, 2020: 4), la ciudad se ha convertido en sala de espera para migrantes en MPP, en condición irregular, deportados y personas que provienen de la frontera sur de México para registrarse en la lista de espera al MPP. Los estragos y afectaciones a las Organizaciones de la Sociedad Civil (OSC) en México, ya se hacían evidentes antes del Covid-19 con la suspensión del Fondo de Apoyo a Migrantes (FAM) cuyos recursos eran utilizados para gastos de operación de las OSC. Lo anterior hace visible la incertidumbre, inestabilidad y vulnerabilidad de la población migrante y de los albergues que los acogen. Por tanto, en los últimos años, la actuación de los organismos internacionales gubernamentales y no gubernamentales resulta fundamental para complementar y apoyar la gestión y atención de los flujos migratorios que se establecen de forma temporal en la ciudad de Mexicali. 


\section{El Covid-19 llega A Baja CAlifornia, MÉxico}

La pandemia del Covid-19 o SARS-CoV-2 declarada el 11 de marzo por la Organización Mundial de la Salud (OMS) trajo consigo la intensificación de las deportaciones de Estados Unidos a las ciudades fronterizas de México, ocurriendo en horas atípicas y en sus inicios con carencia de protocolos de recepción del lado mexicano (Moreno y Olvera, 2020). Se suma a este escenario, la vulnerabilidad a la que se enfrentan mujeres, niñas y comunidad LGBTTTIQ+, que viven experiencias y retos migratorios diferentes a los hombres, haciéndoles proclives por el confinamiento propiciado por Covid-19 a situaciones de violencia sexual basada en género tanto en hogares como en albergues (ONU Mujeres México, 2020).

Ante la emergencia sanitaria, el gobierno del estado de Baja California, (2020), siguió las medidas preventivas anunciadas a nivel federal, que implicaron la suspensión de actividades no esenciales y lineamientos para prevenir y mitigar el Covid-19 (DOF, 2020). Sin embargo, el tránsito transfronterizo que se da por cuestiones laborales, turísticas, sociales y familiares, producen cruces continuos por las fronteras Mexicali-Calexico y por su proximidad Tijuana-San Diego, que incentivaron el número de contagios, posicionando a $\mathrm{BC}$ en los primeros lugares en casos positivos acumulados. En este contexto, la Comisión Mexicana de Ayuda a Refugiados (COMAR) cerró sus puertas y en Estados Unidos se han diferido las citas para MPP, dejando a la deriva y en desamparo a las personas migrantes en sus procesos migratorios, en algunos casos por el vencimiento de permisos incurren en un estatus migratorio irregular, que les limita el acceso a servicios básicos de salud que no son Covid-19, también han presentado afectaciones en su salud mental y en la mayoría de los casos perdieron su empleo que les permitía cubrir algunos alimentos y estancia en los albergues.

En este contexto, es muy importante hacer un recuento de la situación que han enfrentado estos albergues operados por la sociedad civil bajacaliforniana a partir de la llegada del Covid-19 a la entidad, en particular por el complejo escenario que enfrentan estas organizaciones ante la suspensión de los fondos gubernamentales, hecho que ha causado que su sostenimiento dependa totalmente de donativos provenientes de la sociedad, fundaciones y organizaciones internacionales así como también por posicionamiento del estado de Baja California como epicentro nacional de la pandemia. 


\section{INDICADORES DE ATENCIÓN A MIGRANTES EN EL CONTEXTO DE Covid-19}

Los albergues son la primera referencia que obtienen los migrantes de retorno y en tránsito sobre un lugar seguro donde puedan dormir, comer, asearse y obtener un cambio de ropa. Según El Colegio de la Frontera Norte (Colef) en Baja California existen cerca de 42 albergues operados por organizaciones de la sociedad civil en cuya responsabilidad ha quedado el tema de la atención de los flujos migratorios (Coubès et al., 2020: 4), 11 de ellos se encuentran en Mexicali.

Al respecto, no existe un protocolo de atención para la operación de estos albergues conducidos por asociaciones civiles definido por parte de la autoridad gubernamental, sin embargo, se han emitido parámetros mínimos bajo los cuáles deben operar para garantizar una óptima atención de los flujos de migrantes en el contexto de la pandemia por Covid-19.

Esta atención se debe prestar bajo el principio de igualdad, no discriminación y equidad en las oportunidades e independiente del estatus migratorio con la finalidad de que los migrantes no caigan o agudicen situaciones de vulnerabilidad.

\section{Monitoreo y acceso a la salud}

Una de las primeras pautas a seguir es el monitoreo y el acceso a la salud, lo anterior se refiere a las prácticas de prevención tales como escaneos para detectar síntomas, realización de pruebas y tener áreas aisladas para los recién llegados a los albergues así lo indica United $\mathrm{Na}$ tions Human Rights Office of the High Commissioner (2020). También El Colef enfatiza la importancia de implementar acciones preventivas dentro de los albergues que incluyen; proporcionar información sobre la enfermedad, pero también sobre el acceso al sistema de salud local a los migrantes, ésta debe ser en un idioma que los migrantes entienden, en un formato accesible y culturalmente apropiada. Al personal que opera en los albergues se le debe capacitar sobre protocolos de identificación de casos, de traslado a lugares destinados para personas con Covid-19 y atender personas con síntomas respiratorios moderados (Coubès et al., 2020). También el Instituto Nacional de Migración (INM) recomienda identificar a las personas migrantes vulnerables frente al Covid-19 (adultos mayores o con una enfermedad crónica), y disponer (para ellos) de áreas de estancia que estén alejadas del paso 
constante o regular al interior de las instalaciones migratorias, para evitar su contagio (INM, 2020).

\section{Logística y funcionamiento}

Lo siguiente es referente a la logística que debe implementarse para una óptima atención de los migrantes ante la pandemia, algunos albergues han adoptado la política de "nadie entra, nadie sale" es decir, que a partir del inicio de la cuarentena no recibieron a más personas y sólo siguieron operando con las que ya estaban instaladas, en algunos no les permiten entradas y salidas, en otros les permiten salir solo a aquellos que deben trabajar y se les permite regresar solo a dormir, al respecto la Secretaría de Salud (SS) recomienda suspender las salidas a la población albergada. Otra medida en la que coinciden la SS y la OIM es en llevar un censo o registro de personas albergadas (OIM, 2020; Secretaría de Salud, 2020).

\section{Instalaciones}

Independientemente de la logística bajo la cual opere el albergue se recomienda; abastecerlos con material de aseo personal, material de limpieza y cubrebocas. Contar con insumos como: jabón, gel desinfectante con base de alcohol y bolsas de basura, pañuelos desechables y cubrebocas y establecer protocolos de sanitización de dormitorios y comedores. Otra medida fundamental reside en evitar el hacinamiento y tratar de mantener medidas de sana distancia (Coubès et al., 2020). El INM recomienda que en los espacios comunes y dormitorios se garantice al menos 1.50 metros de distancia entre camas, y la posición intercalada al dormir, así como colocar cortinas de tela o material de fácil limpieza entre cama y cama, en el caso de la SS recomienda dos metros de distancia entre camas. Respecto de baños y regaderas la SS y OIM coinciden en recomendar que sean separadas para hombres y mujeres y deben existir al menos un baño por cada 30 personas máximo (INM, 2020; OIM, 2020; Secretaría de Salud, 2020).

Adicional al esfuerzo y operación interna de los propios albergues ante la crisis sanitaria, se suman a las OSC, fundaciones y organizaciones internacionales que coadyuvan con los albergues para mejorar las condiciones de éstos y ofrecer una mejor atención a las personas migrantes que son alojadas. De tal manera, que la actuación de estas organizaciones es diversa y tiene cabida en las dimensiones ya seña- 
ladas: 1) monitoreo y acceso a la salud; 2) logística y funcionamiento e 3) instalaciones.

\section{Metodología}

El estudio se realiza desde un enfoque mixto, es decir cualitativo y cuantitativo (Sampieri, Fernández y Baptista, 2010), que permite recolectar, analizar e interpretar datos e información de las acciones que se llevan a cabo en albergues de la ciudad y de las organizaciones que les coadyuvan en el marco de la pandemia de Covid-19 durante el año 2020 en Mexicali, Baja California.

La estrategia metodológica que se siguió consistió en: i) la revisión documental y análisis de indicadores que refieren a los parámetros mínimos de operación que deben ser adoptados por los albergues ante la pandemia de Covid-19. Se identifican tres clasificaciones de indicadores (ver Tabla 1); ii) el trabajo de campo consistió en la aplicación de entrevistas a encargados de albergues y colaboradores de organizaciones y; iii) se realizó análisis estadístico descriptivo, así como procesamiento de la información mediante Atlas ti (Varguillas, 2006), para obtener asociaciones entre la información obtenida y los indicadores señalados previamente.

A partir de los indicadores identificados, se diseñaron dos cuestionarios de entrevista semi abiertos que permitieron reconocer aspectos de interés (López-Roldán y Fachelli, 2015). Un cuestionario es dirigido a los albergues y otro a las organizaciones, ambos instrumentos se estructuran en tres secciones que refieren a los tipos de indicadores y contienen preguntas abiertas y cerradas de opción múltiple. El método de selección de casos fue mediante muestreo no probabilístico discrecional y bola de nieve (Goldberg, 2020; Pérez y Pesántez, 2017). Según los 11 albergues para migrantes que existen en Mexicali (Coubès et al., 2020) y debido a la situación generada por Covid-19, tres han cerrado, uno opera parcialmente y siete continúan brindando servicios con restricción de nuevos ingresos de personas migrantes. Estos últimos se tomaron como referencia para conformar una muestra de cinco albergues. De acuerdo con las recomendaciones para mantener el distanciamiento social (Banerjee y Nayak, 2020), las entrevistas que se realizaron a encargados de los albergues fueron cuatro, vía telefónica, y una presencial (ver Tabla 1). Con respecto a las organizaciones (ver Tabla 2), se realizaron tres entrevistas a colaboradores vía telefónica. 
Tabla 1. Clasificación de indicadores

\begin{tabular}{|c|c|}
\hline \multicolumn{2}{|c|}{ Clasificación 1. Monitoreo y acceso a la salud } \\
\hline Categorías & Indicadores \\
\hline \multirow[t]{5}{*}{ 1.- Acciones de prevención } & 1.1 Realización de pruebas. \\
\hline & 1.2 Designación de áreas para aislamiento. \\
\hline & 1.3 Escaneos para detectar síntomas. \\
\hline & 1.4 Brindar información sobre la enfermedad. \\
\hline & $\begin{array}{l}\text { 1.5 Proporcionar información sobre el acceso al sistema de } \\
\text { salud local a los migrantes, en un idioma que los migran- } \\
\text { tes entienden, en un formato accesible y culturalmente } \\
\text { apropiada. }\end{array}$ \\
\hline \multirow[t]{3}{*}{ 2.- Capacitación } & $\begin{array}{l}2.1 \text { Dirigida al personal, sobre protocolos de identificación } \\
\text { de casos Covid-19. }\end{array}$ \\
\hline & $\begin{array}{l}2.2 \text { De traslado a lugares destinados para personas con Co- } \\
\text { vid-19. }\end{array}$ \\
\hline & $\begin{array}{l}2.3 \text { Atención de personas con síntomas respiratorios mode- } \\
\text { rados. }\end{array}$ \\
\hline 3.- Personas vulnerables & $\begin{array}{l}\text { 3.1 Identificación de las personas migrantes vulnerables } \\
\text { frente al Covid-19 (adultos mayores o con una enfermedad } \\
\text { crónica), y disponer (para ellos) de áreas de estancia que } \\
\text { estén alejadas del paso constante o regular al interior de las } \\
\text { instalaciones migratorias. }\end{array}$ \\
\hline \multicolumn{2}{|c|}{ Clasificación 2. Logística y funcionamiento } \\
\hline Categorías & Indicadores \\
\hline \multirow{3}{*}{$\begin{array}{l}\text { 4.- Ingresos y salidas de perso- } \\
\text { nas migrantes en los albergues }\end{array}$} & 4.1 No permiten nuevos ingresos. \\
\hline & $\begin{array}{l}\text { 4.2 Siguen operando los albergues con las personas migran- } \\
\text { tes que se alojaron desde antes de la pandemia de Covid- } 19 \text {. }\end{array}$ \\
\hline & 4.3 Se permiten salidas solo a quienes van a trabajar. \\
\hline 5.- Censo o registro diario & 5.1 Identificar la periodicidad de los registros. \\
\hline \multicolumn{2}{|r|}{ Clasificación 3. Instalaciones } \\
\hline Categorías & Indicadores \\
\hline $\begin{array}{l}\text { 6.-Abastecimiento de materia- } \\
\text { les }\end{array}$ & $\begin{array}{l}\text { El material con el que cuenta el albergue es suficientes, es- } \\
\text { caso o no tiene: } \\
\text { 6.1 Material de aseo personal. } \\
\text { 6.2 Material de limpieza. } \\
\text { 6.3 Cubrebocas. } \\
\text { 6.4 Material de aseo (jabón, gel desinfectante con base de } \\
\text { alcohol y bolsas de basura). } \\
\text { 6.5 Pañuelos desechables. }\end{array}$ \\
\hline \multirow[t]{3}{*}{ 7.-Protocolos de sanitización } & 7.1 Se realiza o no en dormitorios. \\
\hline & 7.2 Se realiza o no en comedores. \\
\hline & 7.3 Se realiza o no en las áreas comunes. \\
\hline 8.-Sana distancia & 8.1 Dormitorios $1.5 \mathrm{~m}$ a $2 \mathrm{~m}$ \\
\hline \multirow[t]{2}{*}{ 9.- Baños y regaderas } & 9.1 Separados Hombres y Mujeres. \\
\hline & 9.21 baño por cada 30 personas máx. \\
\hline
\end{tabular}

Fuente: elaboración propia. 
Tabla 2. Lista de albergues entrevistados

\begin{tabular}{clcc}
\hline Número & \multicolumn{1}{c}{ Albergue } & Iniciales & Fecha \\
\hline 1 & Hijo Pródigo & HP & $4 / 08 / 2020$ \\
2 & El Camino a un Nuevo Amanecer & CNA & $28 / 07 / 2020$ \\
3 & La Posada del Migrante & PM & $31 / 07 / 2020$ \\
4 & Casa de Ayuda Alfa y Omega & A\&O & $31 / 07 / 2020$ \\
5 & Hotel del Migrante & HM & $28 / 07 / 2020$ \\
\hline
\end{tabular}

Fuente: elaboración propia.

Tabla 3. Lista de organizaciones consideradas en las entrevistas

\begin{tabular}{clcc}
\hline Número & \multicolumn{1}{c}{ Organización } & Iniciales & Fecha \\
\hline \multirow{2}{*}{1} & Organización Internacional & OIM & $29 / 07 / 2020$ \\
& para las Migraciones & & $03 / 08 / 2020$ \\
2 & Border Kindness & BK & $24 / 06 / 2020$ \\
3 & Médicos Sin Fronteras & MSF & $4 / 08 / 2020$ \\
\hline
\end{tabular}

Fuente: elaboración propia.

\section{ANÁLISIS DE RESULTADOS}

\section{Acciones implementadas en los albergues}

Monitoreo y acceso a la salud

De las acciones de prevención identificadas, en cuatro albergues se observó que éstas son similares: i) se toma la temperatura corporal, se realiza detección de síntomas tempranos y existe un área especial para posibles casos de Covid-19; ii) en tres de ellos, estas acciones son diarias, incluso dos veces al día. En el cuarto albergue, se mencionó que son esporádicas, debido a que las personas salen y entran del lugar en diferentes horarios, también se les comenta a las personas migrantes que si llegan a tener algún síntoma se comuniquen con el encargado. En el quinto albergue, se encontró que las acciones de prevención mencionadas no se llevan a cabo, tampoco se cuenta con un área de aislamiento, únicamente se les proporciona gel en cuanto ingresan las personas y se les solicita el lavado de manos.

La información que se brinda a las personas migrantes dentro de los albergues con respecto a la prevención, detección de síntomas y posibles casos de Covid-19, es proporcionada por encargados de los 
albergues y en gran medida por organizaciones como OIM, ACNUR, MSF, médicos del Instituto Mexicano del Seguro Social (IMSS) y algunos médicos voluntarios. La información y capacitación que ofrecen las organizaciones e instituciones a los albergues, han sido presencial y en línea, también se han proporcionado materiales impresos de diferentes tipos.

\section{Logística y funcionamiento}

La crisis sanitaria por Covid-19, ha ocasionado que varios albergues se vean en la necesidad de limitar el ingreso de nuevas personas para no poner en riesgo a la población que ahí se encuentra y mantener en la medida de lo posible las condiciones que se requieren para la sana distancia. De los cinco albergues con respecto a la recepción de nuevos ingresos de personas migrantes, se identificó que en HP y HM siguen recibiendo personas, debido a que el flujo migratorio se mantiene y argumentan que es necesario abrirles las puertas para evitar la situación de calle. Las medidas que se siguen para regular los nuevos ingresos de personas migrantes son variadas, van desde las más sencillas como es el caso de un albergue que realiza detección de síntomas y aplicación de un cuestionario, en otro albergue se mantienen en un área de aislamiento preventivo en carpas proporcionadas por la OIM. En PM en casos especiales o extremos recibe personas, sin embargo, es necesario aislarlas en otro lugar alrededor de 25 días para incorporarlas posteriormente al albergue. En CNA y A\&O no han recibido personas migrantes desde que comenzó la cuarentena por Covid-19.

A pesar de las restricciones de los nuevos ingresos, en los cinco albergues se permiten salidas por cuestiones de trabajo o por algún asunto en particular. De manera generalizada hacen hincapié a las personas de no desviarse durante su traslado, en las medidas de prevención y uso de cubrebocas. Sin embargo, en HP y PM, indicaron que una vez que regresan al albergue se les toma la temperatura y se les proporciona gel y solo en HP, se lleva un registro diario de la temperatura de cada persona en un cuaderno y se anota si presentan algún síntoma.

Además de las medidas de prevención por Covid-19, se realizan conteos y registros diarios de las personas migrantes en tres albergues, y en dos mencionaron que es cada fin de semana, debido a que en estos días se reducen las salidas por cuestiones laborales o de otra índole y se encuentra la mayor parte de las personas. 


\section{Instalaciones}

Respecto a los protocolos de sanitización, es necesario aclarar que no son estandarizados ni se aplican las mismas medidas en todos los albergues, sino que cada uno con sus propios medios y recursos realizan acciones relativas a la limpieza y desinfección de espacios. En CNA y $\mathrm{A} \& 0$, dos veces por semana se desinfecta con una máquina que se comparte y que fue adquirida por ellos, la cual es utilizada para dormitorios, áreas de comedor y comunes. En HP y PM se realiza limpieza diariamente en todas las áreas. En HM, la sanitización se realizó en la parte de afuera gracias al apoyo de una organización, aunque ellos no realizan propiamente esta actividad, sino que las personas albergadas son quienes hacen la limpieza de su espacio.

Como medida adoptada para preservar la sana distancia, en tres albergues las literas, camas o colchonetas se encuentran a 1.5 metros de distancia y en posición intercalada, en un cuarto albergue, la distancia coincide con la mencionada, aunque el acomodo es en paralelo y en el quinto albergue, la distancia es de un metro con otro tipo de acomodo. Cabe mencionar que en ninguno de los cinco albergues se han colocado cortinas o material de fácil limpieza entre cama y cama.

En relación a la suficiencia y conveniencia según las recomendaciones de autoridades y organizaciones, con respecto a los baños y regaderas en proporción de una por cada 30 personas como máximo, se observó que por el número de personas alojadas hasta el día en que se realizó la entrevista, cuatro cumplían con este aspecto y una ligeramente sobrepasado, sin embargo, fueron cuatro de cinco albergues, los que tienen división para hombres y mujeres.

De gran relevancia, han sido en estos momentos de crisis sanitaria los materiales con los que cuentan los albergues para mantener las condiciones de higiene y limpieza de las personas alojadas y de las instalaciones en general. Con respecto a los materiales de aseo personal, de limpieza general y cubrebocas cuatro indicaron suficiencia, el quinto albergue indicó escasez. Para el material de aseo como jabón, gel desinfectante con base alcohol y bolsas de basura, pañuelos desechables indicaron escasez en tres de cinco albergues. 


\section{ACCIONES DE ORGANIZACIONES}

\section{Médicos sin fronteras}

En cuanto a las acciones de prevención entregan material informativo, insumos de higiene como guantes, gel, jabón, lavamanos portátiles, implementos generales de limpieza e higiene. Asimismo, se han realizado campañas informativas mediante la entrega de folletos, y material para prevención, así como capacitaciones.

Respecto a la capacitación a pesar de que cerraron el centro de atención en Mexicali, ofrecen el servicio de capacitación y prevención del Covid-19, han estado asistiendo a los albergues para realizar trabajo de capacitación e identificación de posibles casos, prevención y aislamiento, así como mostrar la forma correcta de desinfectar las instalaciones, la periodicidad es de acuerdo con las necesidades.

Esta organización no ha sugerido en concreto un protocolo de atención dentro de los albergues, sino que les da sugerencias sobre las mejores prácticas y los encargados de albergues deciden de forma directa si deben o no aplicarse, se mandan también materiales de apoyo con información.

\section{Border Kindness}

En esta organización tienen un consultorio ubicado en la zona centro de Mexicali, hasta ahí llegaron dos casos de mujeres migrantes haitianas embarazadas que presentaron Covid-19 pero asintomáticas, siguen trabajando en monitoreo, pero no en albergues directamente sino en las instalaciones del consultorio.

\section{Organización Internacional para las Migraciones}

La OIM es una de las organizaciones que ha proporcionado mayor apoyo a los albergues, en el área de prevención se documentaron acciones como: donativos de litros de gel antibacterial, jabón líquido para manos, paracetamol, mascarillas todo lo que implica un tema de respuesta Covid-19, en conjunto con ACNUR han implementado lo que llaman respuesta wash que es del inglés; agua, sanitización e higiene éstos son implementos para la limpieza personal como lavabos portátiles. A través de la Organización Mundial de la Salud (OMS) y la Organización Panamericana de la Salud (OPS) se ha capacitado a los albergues y se les ha enviado manuales, documentos guía, se han realizado webinars 
dirigido a los encargados de los albergues para que realicen lo propio en el manejo de éstos, como respuesta Covid-19 y como ruta se ha platicado con ellos para el deber ser (aunque se sabe que no están actuando los de la jurisdicción sanitaria de gobierno). OIM ha dado pláticas sobre medidas de prevención de Covid-19, detección de síntomas, manejo de casos y medidas de prevención. Se han donado manuales, trípticos, folletos, carteles, impresos de diferentes tamaños enfocados a la prevención y lo que es Covid-19.

También OIM ha donado carpas de aislamiento a algunos albergues y faltan donar aires acondicionados para que las carpas puedan ser habilitadas al 100 por ciento, se está construyendo otra ruta; el hotel filtro, ${ }^{1}$ otros albergues ya saben de esto, para que los manden al hotel rentado por OIM, ahí estarán en cuarentena y después con certificado médico se mandarán a los albergues que hayan realizado un convenio con OIM para estos fines.

Asimismo, han apoyado con el pago para realizar pruebas de Covid-19 en laboratorios privados para casos sospechosos, en este caso se ha tenido que recurrir a realizarlas así ya que en las instancias de salud pública no se aplica una suficiente cantidad de pruebas

Respecto de las instalaciones e insumos el apoyo principal de esta organización ha consistido en que directamente no se brinda a los albergues la desinfección, pero como respuesta se donaron materiales a los que pueden tener acceso de manera cotidiana, como cloro, se les dio la fórmula desinfectante, así como conocimiento de la cantidad que pueden preparar y que ellos puedan utilizar para desinfectar, se les dio el Know How para que ellos lo pudieran hacer.

También se han donado kits de ropa de cama, mascarillas, insumos de higiene personal, dispensadores de gel, jabón, cloro, también se activó plan de respuesta Covid-19 en siete albergues de Mexicali para brindarles tarjetas de alimentos, con montos de varios cientos de miles de pesos, la duración de este apoyo es de ocho semanas cuando menos, sólo para alimentación en albergues.

1 La OIM en colaboración con otros actores públicos y privados ha establecido en otras entidades federativas fronterizas como Ciudad Juárez y Tijuana hoteles filtro, donde los migrantes permanecen en él hasta dos semanas bajo supervisión médica antes de acceder a alguno de los albergues de estas localidades. Próximamente se planea abrir uno en Mexicali. 


\section{CONCLUSIONES Y RECOMENDACIONES}

La situación de abandono por parte del gobierno federal y estatal hacia las organizaciones de la sociedad civil no se suscitó específicamente a raíz de la pandemia, sino que, viene derivada de una política de austeridad del gobierno actual en donde se opta por un recorte de recursos dirigidos a temas que ordinariamente eran atendidos por estos organismos con aporte de recursos públicos, uno de ellos es el tema de la migración. En este caso, las afectaciones iniciaron a principios del año 2019, y se agudizaron aún más con la llegada de las caravanas de migrantes centroamericanos y el incremento de los flujos de migrantes en tránsito de otras nacionalidades y migrantes de retorno, es decir deportados.

Desde entonces, estas organizaciones han tenido que depender de los incipientes apoyos en especie que les brinda el gobierno a través de la federación y los estados (entidades federativas), siendo su principal sustento los donativos y aportes que reciben de los organismos internacionales gubernamentales y no gubernamentales, fundaciones, instituciones académicas y población en general.

La situación a raíz de la pandemia de Covid-19 no ha sido diferente para estos albergues como se ha evidenciado en este artículo, el manejo de los recintos para migrantes han carecido de una atención por parte de las instancias gubernamentales que se ha traducido en falta de apoyo para la implementación de protocolos y el abastecimiento de insumos, situación que ha tenido como consecuencias que algunos han tenido que dejar de operar de forma temporal y otros han restringido su funcionamiento, a pesar de que los flujos migratorios no han experimentado una disminución en el contexto de la pandemia.

Estos albergues, ya estaban padeciendo los efectos de la falta de apoyo por parte del gobierno de México. Ahora enfrentan la crisis sanitaria generada por Covid-19 mediante acciones para su contención y prevención, que en buena parte son definidas por los propios encargados de albergues considerando sus recursos disponibles. La ayuda que reciben de las organizaciones ha sido valiosa, aunque no suficiente para sobrevivir a los estragos de la pandemia, en ese sentido, resulta muy importante la participación de los organismos internacionales en la gestión y atención de los migrantes a pesar de que, para la ciudad de Mexicali, su presencia es relativamente reciente. Como se evidenció en este artículo OIM, ACNUR y Médicos sin Fronteras son los principales 
actores que coadyuvan con los Organismos de la Sociedad Civil que operan los albergues para hacer frente a la contingencia sanitaria con las personas en contexto de migración.

Sin embargo, aún es necesaria la participación y coordinación de los tres órdenes de gobierno, para que en primera instancia se genere la voluntad política, hasta ahora ausente y desplegar protocolos, así como acciones definidas con respecto a Covid-19 en los albergues que atienden personas en contexto de movilidad.

Como segundo punto, establecer acciones coordinadas y articuladas entre las autoridades públicas competentes, encargados de las OSC y con las organizaciones e instituciones que apoyan a la población migrante, mediante mecanismos de colaboración que impliquen un apoyo directo a la red de albergues migrantes y se genere con ello un mayor impacto y alcance para: i) hacer llegar información clara, oportuna y en un idioma entendible, mediante diferentes medios; ii) llevar a cabo un programa de capacitación unificado formal e integral para temas de prevención, tratamiento y actuación con respecto a Covid-19; iii) establecer protocolos estandarizados, claros y bien definidos para desinfectar espacios; iv) definir protocolos de actuación para casos sospechosos o identificados con Covid-19; v) realizar un monitoreo de capacidad de atención en los albergues para canalizar nuevos ingresos y asegurar las condiciones de sana distancia; vi) mantener continuo abastecimiento de materiales que se requieren para limpieza general, sanitización de espacios y aseo personal y; vii) crear un sistema de registro de personas migrantes y encargados albergues que han padecido Covid-19 para medidas de control y futuras estrategias.

Claramente, se requiere del involucramiento y actuación colaborativa de todos los actores para implementar estrategias que contribuyan a una mejor atención en los diferentes albergues, no solo por los efectos propios derivados de la pandemia, sino por la deuda adquirida derivada de los apoyos omitidos a las OSC, porque la población migrante es quién enfrenta y vive los riesgos que la condición de vulnerabilidad le confiere.

\section{REFERENCIAS BIBLIOGRÁFICAS}

Albicker, Sandra, Bojórquez, Ietza, Contreras, Oscar, Coubés, Marie-Laure, Hernández, Alberto, Hernández, Rafael, París, Dolores, Pérez, Gabriel, Ruíz, Olivia y Velasco, Laura (2019). La caravana de migrantes centroamericanos en Tijuana 2018-2019 (Segunda etapa). [Fecha de consulta 30/07/2020]. Disponible en https://www.colef.mx/estudiosdeelcolef/ 
la-caravana-de-migrantes-centroamericanos-en-tijuana-2018-2019-segunda-etapa/

Avendaño, Rosa, Siu, Esmeralda, Romero, Karen y Moreno, José. (2019). Entre Muros: Solicitantes de asilo bajo los Protocolos de Protección a Migrantes (p. 59). Tijuana, B.C: American Friends Service Committee.

Banerjee, Tannista y Nayak, Arnab (2020). "U. S. county level analysis to determine If social distancing slowed the spread of COVID-19", en Rev Panam Salud Pública, 44, 1-7. doi:10.26633/RPSP.2020.90

Bojórquez, Ietza, Peña, Jesús, Hernández, Carlos, Calva, Luis y Zambrano, Elsa (2019). Emif Norte. Informe Anual de Resultados 2018. El Colegio de la Frontera (COLEF). [Fecha de consulta 21/07/2020]. Disponible en https://www.colef.mx/emif/datasets/informes/norte/2018/Emif Norte Informe Anual 2018.pdf

Carrasco, Gonzalo (2013). "La migración centroamericana en su tránsito por México hacia los Estados Unidos”, en Alegatos, (83), 170-194.

Coubès, Marie-Laure; Velasco, Laura; Contreras, Oscar (2020). Migrantes en albergues en las ciudades fronterizas del Norte de México (p. 23). [Fecha de consulta 20/07/2020]. Disponible en https://www.colef.mx/estudiosdeelcolef/migrantes-en-albergues-en-las-ciudades-fronterizas-del-norte-de-mexico/

Devia, Camilo; Ortega, Dina y Magallanes, Marcela (2014). "Violencia luego de la paz: escenarios de posconflicto en Centroamérica", en Revista Republicana, (17), 119-148

DOF. Acuerdo por el que se establecen acciones extraordinarias para atender la emergencia sanitaria generada por el virus SARS-CoV2 (2020).

Equipo Regional de Monitoreo y Análisis de y Centroamérica, D. H. (2019). Informe sobre derechos humanos y conflictividad en Centroamérica 2018 2019. El Salvador: Equipo Regional de Monitoreo y Análisis de Derechos Humanos en Centroamérica.

Gobierno del Estado de Baja California (2020). Rígidas medidas coercitivas impone gobernador Jaime Bonilla Valdez para evitar riesgosa "curva" del Covid-19 en BC. [Fecha de consulta el 30/07/2020]. Disponible en http:// www.bajacalifornia.gob.mx/noticia_gobbc.html?Noticiald=616

Goldberg, Alejandro (2020). "Refugiados en Portugal y procesos de inserción en la universidad: una indagación etnográfica”, en Estudios Fronterizos, 21, 1-21. doi:10.21670/ref.2008050

Instituto Nacional de Migración (2020). Protocolo Covid-19. [Fecha de consulta el 30/07/2020]. Disponible e: https://www.gob.mx/inm/prensa/protocolo-Covid-19?idiom=es

Jiménez, E. (2016). "La violencia en el Triángulo Norte de Centroamérica: una realidad que genera desplazamiento", en Papel Político, 21(1), 167196 
López-Roldán, Pedro y Fachelli, Sandra (2015). Metodología de la Investigación Social Cuantitativa. Bellaterra: Universitat Autònoma de Barcelona. [Fecha de consulta 22/07/2020]. Disponible en https://ddd.uab.cat/ record/129382

Luna, Kausha (2017). Many Haitians Choosing to Stay in Mexico. Retrieved July 23, 2017, from Center for Immigration Studies. [Fecha de consulta el 23/07/2020]. Disponible en https://cis.org/Luna/Many-Haitians-Choosing-Stay-Mexico

Martínez, Graciela, Cobo, Salvador D. y Narváez, Juan C. (2015). “Trazando rutas de la migración de tránsito irregular o no documentada por México", en Perfiles Latinoamericanos, 23(45), 127-155. doi:10.18504/pl2345127-2015

Moreno, José y Olvera, Paulina (2020). Poblaciones migrantes y refugiadas en el contexto de la pandemia Covid-19. [Recuperado 21/07/2020]. Disponible en https://www.youtube.com/watch?v=50xDtA5AfPM

OIM (2020). La Covid-19 y la detención de migrantes: ¿qué pueden hacer los gobiernos y otros actores claves? [web]. Actualización 29 de abril de 2020. [Fecha de consulta el 20/07/2020]. Disponible en https://www.iom.int/ es/news/la-Covid-19-y-la-detencion-de-migrantes-que-pueden-hacerlos-gobiernos-y-otros-actores-claves

ONU Mujeres México (2020). Mujeres migrantes y refugiadas en el contexto de la Covid-19. [Fecha de consulta el 31/07/ 2020]. Disponible en https://mexico.unwomen.org/es/digiteca/publicaciones/2020-nuevo/ abril-2020/mujeres-migrantes-y-refugiadas-en-el-contexto-de-la-covid19

Paris, Dolores (2018). Migrantes Haitianos y Centroamericanos en Tijuana, Baja California, 2016-2017. Políticas gubernamentales y acciones de la sociedad civil. [Fecha de consulta 30/07/2020]. Disponible en http:// informe.cndh.org.mx//images/uploads/menus/40101/content/files/InformeMigrantes20162017.pdf

París, Dolores y García, Adriana (2019). Datos sobre Protocolo de Protección a Migrantes (MPP) "Quédate en México", enero-agosto 2019, en La Gaceta Migratoria, 7. [Fecha de consulta el 20/07/2020]. Disponible en https://observatoriocolef.org/boletin/gaceta_observacoles_mpp/

Pérez, María y Pesántez, Blanca (2017). "Impacto migratorio en las aspiraciones y expectativas educativas y de movilidad social de jóvenes Sigseños", en Migraciones Internacionales, 9(2), 57-84. doi: 10.17428/rmi. v9i33.242

Ruiz, Ana, Tenison, Ethan y Weyandt, Raymond (2020). Las políticas migratorias de Andrés Manuel López Obrador en México (S. Leutert, ed.). [Fecha de consulta 21/07/2020]. Disponible en https://www.strausscenter. org/wp-content/uploads/PRP-216_Las-Políticas-Migratorias-de-Andrés-Manuel-López-Obrador-en-México.pdf 
Sampieri, Roberto, Férnandez, Carlos y Baptista, Pilar (2010). Metodología de la Investigación. México: McGrawHill.

Secretaría de Salud (2020). Recomendaciones sanitarias para refugios temporales y centros de aislamiento voluntario (CAV) en el contexto de Covid-19. [Fecha de consulta 31/07/2020]. Disponible en https://coronavirus.gob.mx/wp-content/uploads/2020/05/Recomendaciones_sanitarias_refugios_temporales_COVID-19.pdf

SEGOB y UPM (2019). Nueva Política Migratoria del Gobierno de México 2018-2024. [Fecha de consulta 1/08/2020]. Disponible en http://portales.segob.gob.mx/es/PoliticaMigratoria/Nueva_Politica_Migratoria

United Nations Human Rights Office of the High Commissioner (2020). Covid-19 y los derechos humanos de los migrantes: guía. Actualización 7 de abril de 2020. [Fecha de consulta 20/07/2020]. Disponible en https:// www.ohchr.org/Documents/Issues/Migration/OHCHRGuidance_COVID19_Migrants_sp.pdf

Urbina, Fernando (2020). Infomex 66620 (p. 3). CDMX.

Varguillas, Carmen (2006). "El uso de atlas.TI y la creatividad del investigador en el análisis cualitativo de contenido upel. Instituto pedagógico rural el mácaro", en Laurus, 12, 73-87. [Fecha de consulta 30/07/2020]. Disponible en https://www.redalyc.org/pdf/761/76109905.pdf

\section{RESUMEN CURRICULAR DE LAS AUTORAS}

Kenia María Ramírez Meda

Profesora investigadora en la Universidad Autónoma de Baja California, Facultad de Ciencias Sociales y Políticas. Doctora en Relaciones Transpacíficas por la Universidad de Colima y Licenciada en Relaciones Internacionales por la UABC, realizó también estudios de posgrado en economía en la Universidad de Castilla la Mancha, España. Autora de diversas publicaciones en revistas indexadas y arbitradas, así como capítulos de libro y ponencias. Miembro del Sistema Nacional de Investigadores (SNI-Conacyt, Nivel 1) Sus publicaciones más recientes versan sobre Estrategias de Integración de Migrantes Haitianos en Mexicali, B.C. en diversos capítulos de libro editados por la UABC, UDG y UABCS.

Dirección electrónica: kenia@uabc.edu.mx

Registro ORCID: https://orcid.org/0000-0001-8813-4422 


\section{Adriana Teresa Moreno Gutiérrez}

Profesora por asignatura en la Facultad de Ciencias Sociales y Políticas de la Universidad Autónoma de Baja California, es egresada de la Maestría en Administración Pública por la misma institución, además posee el grado de Maestría en Ciencias de la Educación y Licenciada en Administración, por la Universidad del Valle de México. Sus líneas de investigación son: migración, vulnerabilidad y políticas públicas.

Dirección electrónica:_teresa.moreno@uabc.edu.mx Registro ORCID: https://orcid.org/0000-0002-3596-6708 